\title{
Método Pilates no Alinhamento Postural de Adultos Revisão Sistemática
}

\author{
Caroline Santos Radmann ${ }^{1}$
}

\begin{abstract}
RESUMO
A postura do corpo humano está relacionada diretamente com a sua saúde, destacando-se que uma má postura ocorre quando há desequilíbrio e compensações musculares, podendo trazer complicações musculoesqueléticas, dores e até problemas respiratórios. Uma forma de prevenir e tratar os desalinhamentos posturais ocorre por meio do método Pilates. Este artigo objetivou analisar os efeitos dos exercícios deste método na postura corporal por meio da análise sistemática de artigos científicos. Este estudo trata-se de uma revisão sistemática da literatura realizada em agosto e setembro de 2018, nas bases de dados SciELO, Lilacs, Medline/Pubmed e PEDro. Seis artigos foram selecionados para participar do estudo entre todos os que foram submetidos aos critérios de inclusão e exclusão e à avaliação por meio da escala Pedro. Dos seis artigos avaliados, apenas dois não encontraram resultados positivos para a melhora do alinhamento postural após a realização de exercícios do método Pilates. Ao analisar os demais artigos, observa-se que, além de encontrarem resultados satisfatórios para o alinhamento postural, expuseram informações que confirmavam as hipóteses de melhora e que complementavam as informações uns dos outros, o que facilitou a interpretação dos resultados encontrados.
\end{abstract}

Palavras-chave: Exercício terapêutico. Postura. Adultos.

\section{PILATES METHOD IN ADULT POSTURAL ALIGNMENT - SYSTEMATIC REVIEW}

\section{ABSTRACT}

The posture of the human body is directly related to its health, where a bad posture occurs when there is imbalance and muscular compensations, being able to bring musculoskeletal complications, pains and even respiratory problems. One way to prevent and treat postural misalignment occurs through the Pilates method. This article aimed to analyze the effects of Pilates exercises on body posture through the systematic analysis of scientific articles. This study is a systematic review of the literature conducted in August and september 2018 in the databases SciELO, Lilacs, Medline/Pubmed and PEDro. Six articles were selected to participate in the study among all those who were submitted to the inclusion and exclusion criteria and to the Pedro scale evaluation. Of the six articles evaluated, only two did not find positive results to improve postural alignment after performing Pilates exercises. When analyzing the other articles, it was observed that, besides finding satisfactory results for the postural alignment, they presented information that confirmed the hypotheses of improvement and that complemented the information of each other, which facilitated the interpretation of the results found.

Keyword: Exercise therapy. Posture. Adult.

Recebido em: 12/4/2018

Alterações requeridas em: $23 / 10 / 2018$

Aceito em: 7/1/2019

\footnotetext{
Graduada em Fisioterapia pela Universidade Estadual do Piauí - Uespi. Pós-graduada em fisioterapia traumato-ortopédica e desportiva pelo Instituto Lato Sensu. Possui formação completa em Pilates e TRX e em auriculoterapia pelo Instituto Brasileiro de Fisioterapia (Ibrafisio). krolradman@hotmail.com
} 


\section{INTRODUÇÃO}

A postura do corpo humano está relacionada diretamente com a sua saúde e é definida como o alinhamento dos segmentos do corpo em relação aos próprios segmentos, ao meio ambiente e em determinado momento. Considera-se boa postura quando há uma sincronia harmoniosa entre as forças impostas, havendo equilíbrio muscular e ausência de compensações. Por outro lado, má postura ocorre quando há desequilíbrio dessa harmonia, podendo trazer complicações musculoesqueléticas, dores e até problemas respiratórios (BANKOFF et al., 2003; FERREIRA, 2013).

Desde a concepção, a vida intrauterina, o nascimento e até a morte, o ser humano passa por uma série de modificações morfológicas, de certo modo padronizadas, influenciadas pela sua genética e pelo ambiente em que se encontra. Em qualquer etapa desse processo evolutivo podem ocorrer estados patológicos ou fatores de distúrbio no controle postural do corpo, como hipocinesia, vícios posturais e alterações posturais, ou então, podem acontecer distúrbios no desenvolvimento simétrico do sistema locomotor, e as desarmonias morfocinéticas que ficarão evidentes e falsearão a mecânica dos movimentos, variando, por exemplo, os pontos de aplicação das forças musculares e a fisiologia da cinesia (BANKOFF et al., 2003).

Adotar uma posição incorreta por muito tempo, por exemplo, sentar de forma inadequada, numa cadeira sem encosto ou repouso para os braços, pode provocar tensões e contraturas musculares, causando adaptações indevidas na postura corporal estática ou dinâmica, consequentemente evoluindo para dor. Uma forma de prevenir e tratar desalinhamentos posturais ocorre por meio do método Pilates (COSTA; ROTH; NORONHA, 2012).

Tradicionalmente tem-se preconizado a importância dos exercícios contínuos para a promoção de saúde e para proporcionar efeitos benéficos sobre a aptidão muscular (força e resistência), metabolismo, função cardiovascular, fatores de risco e bem-estar (POLLOCK et al., 2000).

Um tipo de exercício muito difundido nos últimos anos tem sido o método Pilates, o qual se baseia na realização de movimentos de forma consciente, controlada e associada à respiração, que favorecem o ganho de flexibilidade e de força muscular, que por sua vez facilitam o alinhamento postural, sendo utilizados tanto para prevenção como reabilitação de desequilíbrios corporais (CONCEIÇÃO; MERGENER, 2012; JUNIOR et al., 2008; COSTA; ROTH; NORONHA, 2012; MARÉS et al., 2012; SOUZA et al., 2013).

O método Pilates vem sendo praticado por grande parte da população. Muitos buscam a solução para suas algias, outros, um melhor alinhamento pos- tural e ainda há os que praticam de forma preventiva. Dessa forma observa-se a necessidade de estudos aprofundados e sistematizados que evidenciem seus efeitos reais. Existe uma certa dificuldade para se encontrar evidências suficientes que apoiam a efetividade de programas de exercícios específicos para o tratamento de alterações posturais. Assim, este artigo teve como objetivo analisar os efeitos do método Pilates na postura corporal por meio da análise sistemática de ensaios clínicos randomizados.

\section{MÉTODOS}

Trata-se de uma revisão sistemática da literatura, realizada em agosto e setembro de 2018, nas bases de dados SciELO, Lilacs, Medline, Pubmed e PEDro. Foram utilizadas as combinações dos descritores postura, Pilates e exercícios, em Inglês e Português e combinados com o operador AND (Posture and Pilates; posture and Exercise Movement Techniques; postura e método Pilates; posture and Pilates method). Não houve delimitação de período de publicação dos artigos, ou seja, a pesquisa abrangeu artigos publicados em qualquer ano.

A busca foi realizada por duas pesquisadoras (CSR e JMAF) e ocorreu em duas etapas: primeiramente, ambas buscaram nas bases, artigos que relacionavam o tema proposto; posteriormente cada pesquisadora realizou a avaliação dos textos por meio da escala Pedro, para obtenção de uma nota, critério final para incluí-los definitivamente no estudo.

Foram incluídos estudos randomizados que abordassem os efeitos do método Pilates no alinhamento postural de adultos e que tivessem escore PEDro maior ou igual a 05. Artigos de revisão, estudos de casos, estudos controlados e quase controlados, artigos que associavam o método Pilates com outro método para o tratamento postural e artigos que retrataram o método Pilates no alinhamento postural de pacientes com patologias neurológicas foram excluídos.

\section{RESULTADOS E DISCUSSÃO}

Com a combinação dos descritores postura, $\mathrm{Pi}$ lates e exercícios, foram encontrados 1.009 artigos, dos quais 296 estavam repetidos. Após a leitura dos títulos, resumos e/ou da metodologia dos artigos selecionados, levando em consideração os critérios de inclusão e exclusão, apenas 6 artigos foram para a etapa seguinte, quando foram avaliados de acordo coma escala Pedro. A busca e seleção dos trabalhos são apresentadas conforme o instrumento PRISMA (Preferred Reporting Items for Systematic Review and Meta-Analyses) na Figura 1. 
Figura 1 - Fluxograma de seleção dos estudos. Teresina, 2018

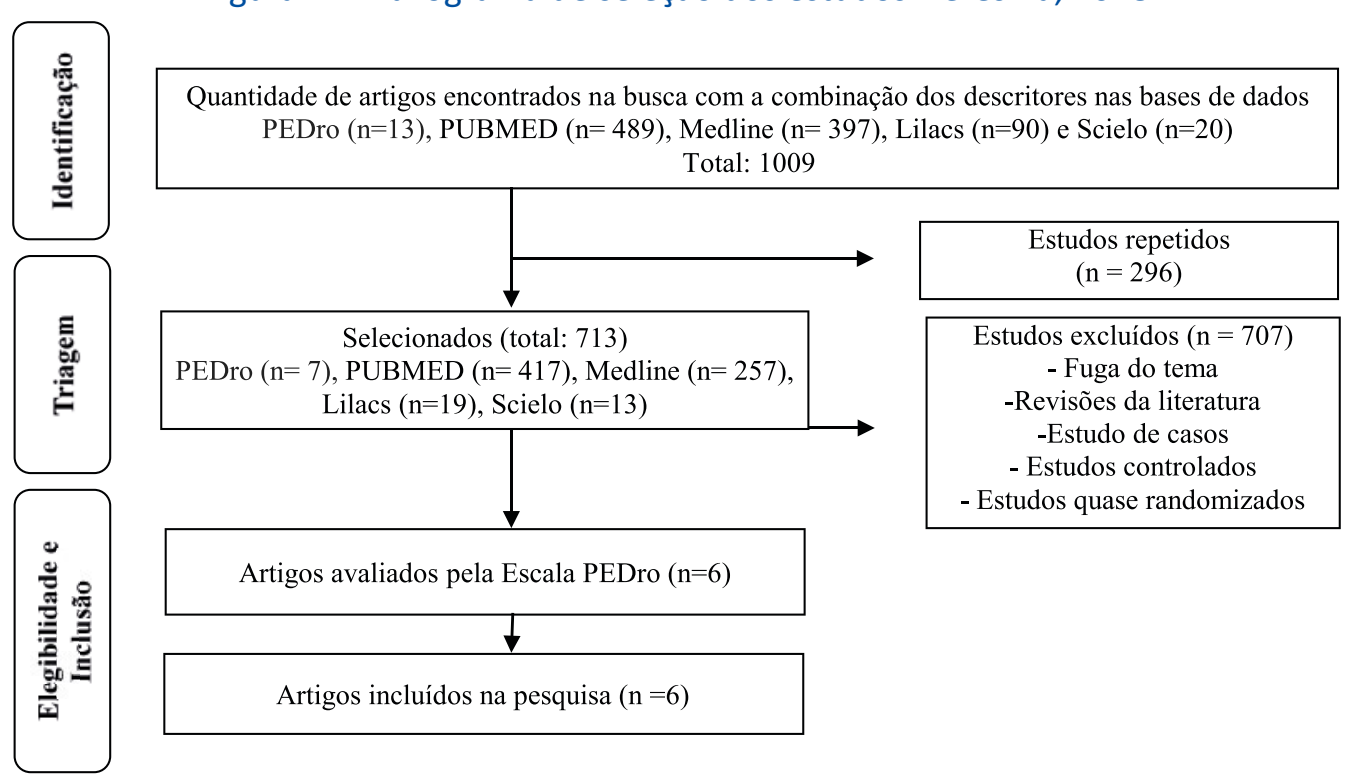

As principais características dos artigos selecionados para compor este estudo encontram-se no Quadro 1.

Quadro 1 - Caracterização dos estudos selecionados para análise. Teresina, 2018

\begin{tabular}{|c|c|c|c|c|c|c|}
\hline AUTOR & $\begin{array}{l}\text { ESCALA } \\
\text { PEDRO }\end{array}$ & OBJETIVO & $\begin{array}{l}\text { TAMANHO DA } \\
\text { AMOSTRA }\end{array}$ & PERFIL DA AMOSTRA & INTERVENÇÃO & DESFECHO \\
\hline $\begin{array}{l}\text { EMERY et al. } \\
\text { (2009) }\end{array}$ & 5 & $\begin{array}{l}\text { Determinar os efeitos do método } \\
\text { Pilates sobre a postura do braço } \\
\text { e do tronco, da força, } \\
\text { flexibilidade e padrões } \\
\text { biomecânicos durante uma } \\
\text { tarefa de flexão do ombro } \\
\text { funcional. }\end{array}$ & 19 & $\begin{array}{l}\text { Adultos jovens, sexo feminino } \\
\text { GE: } n=10 \\
\quad 33,1 \pm 8,6 \text { anos } \\
\text { GC: } n=9 \\
\quad 28,6 \pm 3,7 \text { anos }\end{array}$ & $\begin{array}{l}\text { GE: exercícios do método Pilates } \\
\text { (solo e aparelho) duas vezes por } \\
\text { semana, } 60 \text { minutos cada, durante } \\
12 \text { semanas; GC: sem intervenção. }\end{array}$ & $\begin{array}{l}\text { Melhora da postura e } \\
\text { alinhamento do tronco } \\
\text { superior e dinâmica da } \\
\text { escápula durante a flexão de } \\
\text { braço; } \\
\text { Melhora na força do core. }\end{array}$ \\
\hline $\begin{array}{l}\text { JUNGES et } \\
\text { al. (2012) }\end{array}$ & 7 & $\begin{array}{l}\text { Avaliar a eficácia do método } \\
\text { Pilates em melhorar a postura e a } \\
\text { flexibilidade em mulheres com } \\
\text { hipercifose. }\end{array}$ & 41 & $\begin{array}{l}\text { Mulheres } \\
59 \pm 9 \text { anos } \\
\text { GE: } n=22 \\
\text { GC: } n=19 \\
\text { (não informa a média de idade por } \\
\text { grupo) }\end{array}$ & $\begin{array}{l}\text { GE: exercício do método Pilates } \\
\text { (solo e aparelhos) duas vezes por } \\
\text { semana, } 60 \text { minutos cada, durante } \\
30 \text { semanas. } \\
\text { GC: sem intervenção. }\end{array}$ & $\begin{array}{l}\text { Melhora na postura e } \\
\text { flexibilidade no subgrupo de } \\
\text { mulheres com mais idade, com } \\
\text { hipercifose. }\end{array}$ \\
\hline $\begin{array}{l}\text { KLOUBEC } \\
(2010)\end{array}$ & 6 & $\begin{array}{l}\text { Determinar os efeitos } \\
\text { do exercício de Pilates na } \\
\text { resistência abdominal, na } \\
\text { flexibilidade, na resistência } \\
\text { muscular, na postura e no } \\
\text { equilíbrio. }\end{array}$ & 22 & $\begin{array}{l}\text { Adultos ambos os sexos (25-65 } \\
\text { anos) } \\
\text { GE: } n=25 \\
\quad \text { de } 26 \text { a } 58 \text { anos } \\
\text { GC: } n=25 \\
\quad \text { de } 30 \text { a } 59 \text { anos }\end{array}$ & $\begin{array}{l}\text { GE: exercícios do método Pilates } \\
\text { (em solo) duas vezes por semana, } \\
60 \text { min cada, durante } 12 \text { semanas. } \\
\text { GC: sem intervenção. }\end{array}$ & $\begin{array}{l}\text { Não houve modificações } \\
\text { significativas da postura. } \\
\text { Melhora da flexibilidade e } \\
\text { resistência muscular. }\end{array}$ \\
\hline $\begin{array}{l}\text { LEE et al. } \\
\text { (2016) }\end{array}$ & 7 & $\begin{array}{l}\text { Comparar os efeitos de Pilates e } \\
\text { um programa de exercícios na } \\
\text { postura da cabeça no ângulo } \\
\text { craniovertebral, bem como na } \\
\text { amplitude de movimento } \\
\text { cervical, na dor e fadiga } \\
\text { muscular em indivíduos com } \\
\text { uma postura de cabeça para a } \\
\text { frente }\end{array}$ & 28 & $\begin{array}{l}\text { Mulheres } \\
\text { GE: } n=14 \\
29.2 \pm 4.3 \text { anos } \\
\text { GC: } n=14 \\
28.9 \pm 5.0 \text { anos }\end{array}$ & $\begin{array}{l}\text { GE: exercício do método Pilates (em } \\
\text { solo, com faixas elásticas) três dias } \\
\text { por semana, } 50 \text { minutos cada, } \\
\text { durante } 10 \text { semanas. } \\
\text { GC: exercícios combinados } \\
\text { (alongamentos e exercícios de } \\
\text { resistência) três dias por semana, } \\
50 \text { minutos cada, durante 10 } \\
\text { semanas. }\end{array}$ & $\begin{array}{l}\text { O GE obteve melhores } \\
\text { resultados na postura da } \\
\text { cabeça e da fadiga muscular. }\end{array}$ \\
\hline
\end{tabular}

Fonte: Elaborado pela autora (2018).

Nota: GE: Grupo Experimental; GC: Grupo Controle. 
Ao analisar o Quadro 1 observa-se que entre os estudos realizados somente três especificaram a correção postural esperada, buscando, por exemplo, a correção da postura da cabeça (LEE et al., 2016), a correção da hipercifose (JUNGES et al., 2012) e a correção da postura do braço e tronco (EMERY et al., 2009) por meio do método Pilates. Os demais avaliaram o efeito do método na postura de modo geral.

Dos seis artigos estudados, apenas dois demonstraram não haver melhora da postura dos participantes voluntários após as séries de exercícios de Pilates. Este resultado pode ser encontrado na pesquisa de Kloubec (2010), ao comparar o grupo experimental com o grupo controle. $\mathrm{O}$ autor justifica seus resultados explicando que, quando os desvios posturais são grandes, o método mostra um efeito positivo na correção do desalinhamento, mas quando a postura está dentro dos limites normais o exercício pode ter um efeito mais embutido, não mostrando assim diferenças significativas. Encontrou em seus resultados, contudo, aumento de altura dos participantes, o que sugere a ocorrência de mudanças estruturais no alinhamento da coluna vertebral. Ele ainda ressalta a escassez de estudos que abordem o efeito do método Pilates sobre a postura de indivíduos em condições normais, fator que confirma os achados deste estudo durante as pesquisas nas bases de dados.

O estudo de Sinzato et al. (2013) também não demonstrou melhora significativa da postura dos indivíduos participantes. $O$ protocolo de treinamento foi caracterizado por 12 exercícios realizados em solo, sendo 6 repetições de cada exercício, tendo uma duração de 60 minutos cada sessão, totalizando 20 atendimentos. $\mathrm{O}$ autor do estudo comparou os seus resultados com outros estudos e verificou que, em um deles, ocorreu resultado positivo do Pilates sobre o alinhamento postural, o que divergiu dos seus achados. Em outro estudo os resultados foram similares aos seus. Neste, observa-se que a frequência semanal do tratamento era de apenas um dia, já no outro estudo a frequência era de três dias semanais, o que pode gerar uma diferença no resultado do tratamento devido à diferença entre os intervalos das aulas de Pilates.

Sinzato et al. (2013) explicam que efeitos posturais podem ser atribuídos aos aumentos de resistência e de força muscular proporcionados pelo método Pilates, que permitem uma melhor relação entre os músculos agonistas e antagonistas relacionados ao equilíbrio postural e articular. Tais afirmativas podem ser observadas pelos achados de outros artigos citados e explicados por eles no estudo. Sinzato et al. (2013) justificam que, por não realizarem em sua avaliação a mensuração da força muscular, houve uma limitação em inferir a relação de causa-efeito relacionada ao alinhamento postural. Para os autores, a reeducação postural parece ser tempo-dependente e envolve a adaptação de estruturas e a conscientização postural do corpo.

Nos demais estudos selecionados verifica-se a eficácia do método Pilates na correção postural dos indivíduos participantes. Entre eles, Emery et al. (2009) analisaram a postura do membro superior de forma estática e dinâmica e a postura do tronco, após o treinamento com exercícios do método Pilates. Com seus achados puderam concluir que tais exercícios melhoram as estratégias bilaterais de compensação de movimentos, reparando a simetria postural e prevenindo lesões durante os movimentos dos membros superiores. Observaram também melhora significativa da cifose torácica, atribuindo este fato ao aumento da força e consciência postural de ombro e aumento da força muscular dos eretores da espinha.

Confirmando os achados de Emery et al. (2010) sobre a postura de tronco, o estudo de Junges et al. (2012) traz resultados satisfatórios dos exercícios de Pilates na redução significativa da cifose torácica, esta medida por meio de exame de Raios-X. Segundo os autores, um programa de exercícios com ênfase no fortalecimento da coluna vertebral é importante para prevenir o desenvolvimento de doenças ou disfunções relacionadas à má postura.

Lee et al. (2016) avaliaram os efeitos do Pilates sobre a postura da cabeça, tendo como base o ângulo craniocervical. Seus resultados foram satisfatórios, demonstrando uma melhora significativa da postura da cabeça em relação ao grupo controle. Os autores afirmam que essa melhora pode ser atribuída ao fortalecimento dos músculos profundos do pescoço, a um melhor recrutamento muscular durante movimentos e posicionamentos da cabeça, principalmente quando se trata de um recrutamento muscular local necessário antes de um movimento global. Eles também explicam o fato de o método permitir aos indivíduos uma melhor consciência postural do corpo, dando a eles a capacidade de organizar sua postura.

Ao avaliar a postura dos seus participantes antes e após o treinamento com o método Pilates, Ferreira et al. (2013) observaram uma melhora no alinhamento frontal de ombro, no alinhamento sagital da cabeça e da pelve. Sua avaliação baseou-se em analisar o alinhamento frontal da coluna toracolombar, do ombro e da pelve, e o alinhamento sagital da cabeça e da pelve. Em seu plano de tratamento deram enfoque à respiração, estabilidade pélvica e escapular, alon- 
gamentos e movimentos da coluna vertebral, com os exercícios sendo realizados em sequência progressiva de dificuldade.

Suas intervenções foram realizadas durante seis meses, porém com três meses Ferreira et al. (2013) já observaram algumas alterações significativas na postura de ombro. Corroborando com Emery et al. (2009), os autores justificam que esses resultados podem ocorrer devido ao dato de os exercícios do método proporcionarem uma melhora da cintura escapular quanto à força e estabilidade, melhorando os padrões desnecessários de movimento e alinhando os segmentos do corpo, resultando em uma melhor postura do ombro. Com seis meses de treino observou-se melhora no alinhamento de quadril e da cabeça, o que segundo os autores é justificado porque, além do fortalecimento muscular, o método promove por meio de estratégias cognitivas, sensações táteis e representação mental do gesto, uma consciência postural, confirmando, assim, os achados de Lee et al. (2016).

Ao analisar as informações de cada artigo no Quadro 1, é possível concluir que os diferentes resultados encontrados podem se justificar pelas diferenças existentes entre as amostras dos estudos e os protocolos de intervenção, levando em consideração que cada indivíduo responde de uma determinada maneira a uma situação, estímulo e intervenção.

\section{CONCLUSÃO}

Dos seis artigos avaliados, apenas dois não encontraram resultados positivos para o alinhamento postural após a realização de exercícios do método Pilates. Os demais mostraram resultados satisfatórios na melhora postural. Ao analisar estes estudos observou-se que os diferentes autores expuseram informações que se complementavam entre si, confirmando as hipóteses de melhora uns dos outros, o que facilitou a interpretação dos resultados encontrados.

Existem poucas evidências conclusivas sobre os efeitos primários e secundários do uso do método Pilates em alterações posturais, bem como em alguns distúrbios correlacionados, tais como o retorno às atividades laborais e à vida diária e melhora da função global da coluna lombar e tronco. Dessa forma torna-se importante a realização de estudos com metodologia criteriosa, que permitam embasar melhor, dando evidências científicas aos benefícios posturais alcançados com o método Pilates.

\section{REFERÊNCIAS}

BANKOFF, A. D. P. et al. Estudo das alterações morfológicas do sistema locomotor: postura corporal $x$ obesidade. Revista da Educação Física/UEM, v. 14, n. 2, p. 41-48, 2003. Disponível em: http://periodicos.uem.br/ojs/index.php/RevEducFis/article/viewFile/ 3468/2474. Acesso em: 19 ago. 2018.

COSTA, L. M. R.; ROTH, A.; NORONHA, M. O método pilates no Brasil: uma revisão de literatura. Arq. Catarin. Med., v. 41, n. 3, p. 87-92. 2012. Disponível em: http://www.acm. org.br/revista/pdf/artigos/1151.pdf. Acesso em: 23 ago. 2018.

CONCEIÇÃO, J. S.; MERGENER, C. R. Eficácia do método pilates no solo em pacientes com lombalgia crônica. Relato de casos. Rev. Dor., São Paulo, v. 13, n. 4, p. 385-388, out./ dez. 2012. Disponível em: http://www.scielo.br/readcube/ epdf.php?doi $=10.1590 / \mathrm{S} 180600132012000400015 \&$ pi$d=S 180600132012000400015 \&$ pdf_path=rdor/v13n4/15. pdf\&lang=pt. Acesso em: 23 ago. 2018.

CRUZ-FERREIRA, A. et al. Does Pilates-Based Exercise Improve Postural Alignment in Adult Women? Women \& Health, [s.I.], v. 53, n. 6, p. 597-611, ago. 2013. Informa UK Limited. http://dx.doi.org/10.1080/03630242.2013.817505 . Available from: http://www. pilatesfisios.it/ricerche pdf/ posturalwomen.pdf. Cited: 19 Aug. 2018

EMERY, K. et al. The effects of a Pilates training program on arm-trunk posture and movement. Clinical Biomechanics, v. 2010 , n. 25 , p. $124-130$, out. 2009. Available from: https://www.ncbi.nlm.nih.gov/pubmed/19879677. Cited: 19 Aug. 2018.

FERREIRA, A. C. et al. Does Pilates-Based Exercise Improve Postural Alignment in Adult Women? Women \& Health, [s.l.], v. 53, n. 6, p. 597-611, Aug. 2013. Informa UK Limited. http://dx.doi.org/10.1080/03630242.2013.817505. Available from: http://www. pilatesfisios.it/ricerche pdf/posturalwomen.pdf. Cited: 19 Aug. 2018.

JUNGES, S. et al. Eficácia do método pilates para a postura e flexibilidade em mulheres com hipercifose; Revista Brasileira Ciência e Movimento, v. 20, n. 1, p. 21-33, 2012. Disponível em: https://portalrevistas.ucb.br/index.php/RBCM/ article/viewFile/2721/2134. Acesso em: 23 ago. 2018.

JUNIOR, P. C. N. et al. Os efeitos do método pilates no alinhamento postural: estudo piloto. Fisioterapia Ser, Rio de Janeiro, v. 3, n. 4, p. 210-215, 2008. Disponível em: http:// www. pilatesfisios.it/ricerche\%20pdf/Os\%20 efeitos\%20 do\%20metodo\%20pilates\%20no\%20alinhamento.pdf. Acesso em: 23 ago. 2018.

KLOUBEC, J. A. Pilates for improvement of muscle endurance, flexibility, balance, and posture. Journal Of Strength And Conditioning Research, v. 24, n. 3, p. 661-667, mar. 2010. Available from: https://www.ncbi.nlm.nih.gov/ pubmed/20145572. Cited: 23 ago. 2018.

LEE, S. M. et al. Clinical effectiveness of a Pilates treatment for forward head postureClinical effectiveness of a Pilates treatment for forward head posture. The Society of Physi- 
cal Therapy Science, Republic of Korea, v. 28, n. 7, p. 2.0092.013, 2016. Available from: http://creativecommons.org/ licenses/by-nc-nd/4.0/. Cited: 23 ago. 2018.

MARÉS, G. A importância da estabilização central no método Pilates: uma revisão sistemática. Fisioter. Mov., Curitiba, v. 25, n. 2 p. 445-451, abr./jun. 2012. Disponível em: http:// www.scielo.br/pdf/fm/v25n2/v25n2a22.pdf. Acesso em: 23 ago. 2018.

POLLOCK, M. L. et al. Resistance Exercise in Individuals With and Without Cardiovascular Disease: Benefits, Rationale, Safety, and Prescription An Advisory From the Committee on Exercise, Rehabilitation, and Prevention, Council on Clinical Cardiology, American Heart Association. Circulation, [s.I.], v. 101, n. 7, p. 828-833, 22 Feb. 2000. Ovid Technologies (Wolters Kluwer Health). http://dx.doi.org/10.1161/01. cir.101.7.828. Available from: http://circ.ahajournals.org/ content/101/7/828. Cited: 23 ago. 2018.
SINZATO, C. R. et al. Efeitos de 20 sessões do método Pilates no alinhamento postural e flexibilidade de mulheres jovens: estudo piloto. Fisioter Pesq., v. 20, n. 2, p. 143-150, 2013. Disponível em: http://www.scielo.br/pdf/fp/v20n2/08.pdf. Acesso em: 23 ago. 2018.

SOUZA, D. C. et al. Efeitos de um Programa de Pilates sobre o equilíbrio de suas praticantes no período pós-menopausa. Revista Kairós Gerontologia, v. 16, n. 2, p. 39-49, 2013. Disponível em: http://revistas.pucsp.br/index.php/kairos/ article/view/17630. Acesso em: 23 ago. 2018. 